\title{
Sharing refuges on arid islands: ecological and social influence on aggregation behaviour of wall geckos
}

\author{
Raquel Vasconcelos ${ }^{\text {Corresp., }}{ }^{1,2}$, Sara Rocha ${ }^{3}$, Xavier Santos ${ }^{1}$ \\ ${ }^{1}$ CIBIO, Centro de Investigação em Biodiversidade e Recursos Genéticos, InBIO Laboratório Associado, Universidade do Porto, Vairão, Portugal \\ 2 IBE, Institute of Evolutionary Biology (CSIC-Universitat Pompeu Fabra), Barcelona, Spain \\ 3 Departamento de Bioquímica, Genética e Inmunología, Facultad de Biología, Universidad de Vigo, Vigo, Spain \\ Corresponding Author: Raquel Vasconcelos \\ Email address: raquel.vasconcelos@cibio.up.pt
}

Background. The extent of social behaviour among reptiles is under-appreciated. Two types of aggregations are recognized in lizards: ecological and social, i.e. related to the attraction to a site or to animals of the same species, respectively. As most lizards are territorial, aggregations increase the probability of aggressive interactions among individuals, a density-dependent behaviour.

Methods. After some spurious observations of aggregation behaviour in the endemic Cabo Verde nocturnal gecko Tarentola substituta, we conducted a field-based study in order to thoroughly characterize it. We sampled 48 transects and 40 10x10m quadrats on São Vicente Island to describe the incidence, size and composition of aggregations and to study the effect of gecko and refuge density, plus refuge quality, on refuge sharing. We hypothesize that when density of animals and scarcity of highquality refuges is higher, lizards have increased probability of aggregating. We also predict a consistent pattern of size and composition of groups (male-female pairs, only one adult male per group) throughout the year if there is a selected behaviour to avoid agonistic interactions, and low thermal advantage to aggregating individuals.

Results. We present one of the first evidences of aggregation for Phyllodactylidae geckos. We found that T. substituta forms aggregations around $30-40 \%$ of the time, and that refuges are almost always shared by a female-male pair, sometimes with a juvenile, probably a mechanism to avoid aggressive interactions. We also observed that refuge sharing is dependent on refuge quality, as medium-large (thermally more stable and positively selected) rocks are shared much more frequently than small ones, but independent of adult sizes. Refuge sharing is also directly related to the density of geckos and inversely related to the density of high-quality refuges. We found no relation between body temperatures of geckos and refuge sharing when controlling the effect of rock/air temperature, suggesting that huddling does not improve thermoregulation.

Discussion. Our results suggest that in this harsh environment (rocks reach $46 \circ \mathrm{C}$ ) aggregation incidence is mainly driven by an ecological factor (scarcity of high-quality refuges) and its intersexual composition by social factors (avoidance of agonistic interactions by males, and possible increased reproductive success of the pair). This study sheds some light on the little explored gecko aggregation behaviour and other studies should follow. 
Sharing refuges on arid islands: ecological and social influence on aggregation behaviour of wall geckos

Raquel Vasconcelos ${ }^{1,2}$, Sara Rocha ${ }^{3}$, Xavier Santos ${ }^{1}$

${ }^{1}$ CIBIO, Centro de Investigação em Biodiversidade e Recursos Genéticos, InBIO Laboratório Associado, Universidade do Porto. Campus Agrário de Vairão, Portugal.

${ }^{2}$ IBE, Institute of Evolutionary Biology (CSIC-Universitat Pompeu Fabra), Passeig Marítim de la Barceloneta, Barcelona, Spain.

${ }^{3}$ Departamento de Bioquímica, Genética e Inmunología, Facultad de Biología, Universidad de Vigo, Spain.

Corresponding Author:

Raquel Vasconcelos ${ }^{1,2}$

Email address: raquel.vasconcelos@cibio.up.pt 


\section{Introduction}

2 Many animal species from a wide range of taxa permanently live within groups or form

3 aggregations during certain phases of their life cycle, or under certain environmental conditions,

4 due to beneficial effects (Mouton, 2011). The benefits and costs of living in groups vary from

5 species to species, the latter generally including competition for food resources, reproductive

6 interference and infanticide, inbreeding depression, and higher likelihood of transmission of

7 diseases, parasites and of attracting potential predators with a stronger scent trail (Alexander,

8 1974; Gardner et al., 2015). Because many species are territorial, as are most lizards,

9 aggregations also increase the likelihood of aggressive interactions among individuals, as agonistic behaviour is density dependent (Wilson, 1975).

In lizards, such as in other animals, two types of aggregations are recognized: ecological and social, i. e., related to the attraction to a site or to conspecifics, respectively (Graves \& Duvall, 1995; Kearney et al., 2001). Ecological aggregations typically occur when individuals are attracted to patches of habitat of limited availability or when there is a clumped spatial distribution of exceptionally high-quality factors such as food, shelter, predator avoidance, thermoregulatory or oviposition opportunities (Graves \& Duvall, 1995; Kearney et al., 2001).

17 Social aggregations, on the other hand, are formed when individuals are attracted to conspecifics

18 in order to directly benefit from predator avoidance by group defence or increased vigilance, increased thermoregulatory or mating opportunities (Graves \& Duvall, 1995; Kearney et al., 2001; Chapple, 2003; Gardner et al., 2015).

In comparison with other vertebrate groups, few references to refuge sharing in reptiles, 
22 as well as little quantitative information, exists on the size and composition of these aggregations

23 (Doody, Burghardt \& Dinets, 2013; Gardner et al., 2015). Chapple (2003) documented social

24 aggregations in a group of Australian scincids and referred to enhanced vigilance against

25 predators as one of the benefits of group membership. Mouton (2011) reviewed the tendency of

26 five South African lizard species to aggregate in refuges and found that they mostly aggregate

27 due to shortage of optimal shelters and/or for anti-predatory effects. This author argues

aggregation behaviour to have resulted in strong male territoriality: adult males attempt to set up

harems and only when aggregations become unmanageably big will more than one male occur in the same group (Mouton, 2011). Anecdotal cases of reptile aggregations have been described in agamids (Panov \& Zykova, 2003), chameleons (Toxopeus, Krujit \& Hillenius 1988), tropidurids (Vitt, Zani \& Caldwell, 1996), sceloporines (Ruby, 1977), xantusiids (Davis et al., 2011), xenosaurids (Lemos-Espinal, Smith \& Ballinger, 1997) and amphisbaenidae (Martín et al., 2011), as well as in geckos (Barry, Shanas \& Brunton, 2014; Gardner et al., 2015). In geckos, there are apparently no general rules for explaining aggregation patterns and their composition. For example, Meyer \& Mouton (2007) showed that Bibron's gecko (Chondrodactylus bibronii)

37 lives in groups composed by one male and up to 15 females and juveniles, although they could not distinguish whether or not aggregation was the result of limited availability of optimal shelters or of conspecific attraction. Social aggregations which allow the control of thermal exchange rates via huddling were reported for thick-tailed geckos Underwoodisaurus milii (Shah, 2002; Shine et al., 2003) and common brown geckos Woodworthia maculatus (Bauer, 1990). Reduction of evaporative water loss was suggested for the western banded gecko

44 \& Espinoza, 2006) where often several males and females can be found together (Greenberg, 
45 1943). Pianka \& Vitt (2003) suggested the existence of long term pair bond aggregations of the 46 small diurnal naked-toed gecko genus Gymnodactylus in southern Brazil. A link with some life-

47 traits such as viviparity, high longevity and late sexual maturation was also noticed in some 48 Gekkota, and Diplodactylidae (Barry, Shanas \& Brunton, 2014; Gardner et al., 2015). These examples demonstrate the existence of few general trends in reptile aggregation.

We aimed to shed some light on the little explored gecko aggregations and the causes of this behaviour. Our study focused on Tarentola substituta Joger, 1984 (Phyllodactylidae), an endemic wall gecko from São Vicente Island, Cabo Verde. This species is a good model to study the effect of density of conspecifics within a species in aggregating behaviour as it is especially abundant in comparison to its relatives in continental Africa and Europe, and to other Cabo Verde endemic geckos (Schleich, 1987; Vasconcelos et al., 2013). This species is strictly nocturnal and carefully selects its diurnal retreat sites (Vasconcelos, Santos \& Carretero, 2012). Interestingly, adults tend to select medium-large sized rocks, scarce but high-quality refuges in respect to thermal properties (they reach lower temperatures because they buffer air and soil temperatures better than small rocks, reducing the danger of reaching lethal body temperatures), whereas juveniles used small rocks more frequently (yet still less than expected based on availability alone), sometimes risking overheating (Vasconcelos, Santos \& Carretero, 2012). This non-optimal microhabitat choice was attributed to scarcity of high-quality refuges, extremely high densities of conspecifics, and lack of competitors (there is no other reptile species on most of the island, contrary to the rest of the archipelago) and of native nocturnal ground-predators (Vasconcelos, Santos \& Carretero, 2012). In a scenario of high population density, low predation pressure, and scarcity of optimal microhabitats (Vasconcelos, Santos \& Carretero, 2012), our study aimed to describe aggregation 
68 patterns (incidence, size and composition of groups in age and sex class) of T. substituta on São

69 Vicente Island, and to test if these are dependent on ecological (e. g quality of the refuge, density

70 of geckos, refuge availability) and/or social factors (e.g. enhanced thermoregulation).

71 Specifically, we have examined whether the tendency to aggregate is mostly related to high-

72 quality refuge scarcity (ecological aggregation) or to improved thermoregulation by physical

73 contact with conspecifics, or increasing mating opportunities (social aggregation). Following the

74 above, we hypothesized that when a lizard population, such as T. substituta, presents high

75 intraspecific density and scarcity of high-quality refuges, has thus increased likelihood of

76 presenting aggregations as predicted by Mouton (2011). Thus, we additionally predict that there

77 will be: i) an inverse correlation between refuge sharing and availability of high-quality refuges

78 or/and gecko density across sampling sites; ii) a consistent pattern of size and composition of

79 groups (male-female pairs and only one adult male) throughout the year - based on the

80 assumption that the male-biased dimorphism displayed by the species is an indication of male

81 territoriality - if there is a selected behaviour to avoid agonistic interactions, and not only during

82 the mating season, or a variable pattern along the year if related to mating; iii) low thermal

83 advantage to aggregating individuals.

84

85 Materials and Methods

86 Study area

87 São Vicente Island belongs to the windward island group of Cabo Verde Republic (Figure 1).

88 The island is volcanic with a landscape dominated by stony plains, sandy dunes and barren hills.

89 Except for the summit of Monte Verde $(774 \mathrm{~m})$, the island is mostly composed of dry habitats

90 with sparse or no vegetation (Figure 1). Due to its volcanic nature, almost no burrows or caves 
91 are present.

92

93

94

95

96

97

98

99

100

101

102

103

104

105

106

107

108

109

110

111

112

113

On São Vicente Island, air temperatures are $27.2 \pm 0.8^{\circ} \mathrm{C}$ on average during the warmest months, between July - October, ranging from 24.4 to $28.6^{\circ} \mathrm{C}$, and $18.9 \pm 0.8^{\circ} \mathrm{C}$ on average during the coolest months, between December - February, ranging only from 15.7 to $20.4{ }^{\circ} \mathrm{C}$ due to the moderating influence of the Atlantic Ocean (Hijmans et al., 2005). Air temperature, Ta, is strongly negatively correlated with humidity (Vasconcelos, Santos \& Carretero, 2012) and so it can be used as its surrogate.

98

\section{Study species}

Tarentola substituta (Phyllodactylidae) is a flattened, robust oviparous gecko with a long tail and a delicate head with relatively long, pointed snout, and with a mean snout-vent length (SVL) of $51.60 \pm 3.64 \mathrm{~mm}$ (Joger, 1984; Vasconcelos et al., 2012). Based on previous work, individuals smaller than $45 \mathrm{~mm}$ SVL were considered juveniles (Vasconcelos, Santos \& Carretero, 2012), as they lacked sexual characters, i. e. ovarian follicles seen by transparency in females, and enlarged hemipeneal spurs and more developed cloacal pouches in males (Vasconcelos et al., 2012). This species also presents marked sexual dimorphism, with adult males presenting, besides the more pronounced cloacal pouches, higher body mass and larger body size than adult females (Atzori et al., 2007; Vasconcelos, Santos \& Carretero, 2012). It is distributed all over the island, but avoiding higher elevations, i.e. colder and more humid areas (Schleich, 1987;

Vasconcelos et al., 2012). A previous study showed that individuals are active throughout the year (Schleich, 1987). Another study found that the species is hidden in refuges during the day, and that only rocks are used as refuge (Vasconcelos, Santos \& Carretero, 2012). No data about the longevity of the species or sister taxa is available. 


\section{Geckos and refuge sampling}

116 We have conducted a field-based study to obtain direct evidence of aggregation behaviour.

117 Geckos and refuges were sampled following two complementary methods: 1) linear transects,

118 performed at a single locality with confirmed gecko presence, and 2) quadrats randomly

119 distributed within the main habitats of the island. These two sampling methods had specific and

120 different objectives: transects were used to test whether specific refuges were frequently shared

121 by more than one animal and if some refuges were shared more often than others according to

122 their quality (see below). To test if this refuge sharing was dependent of the densities of geckos

123 or refuge availability, the quadrat methodology was applied. The original raw data are available

124 in Supplemental Data S1.

125 Transects were performed on the north-western side of the island, approximately $5 \mathrm{~km}$

126 southwest of the island capital, Mindelo (Figure 1) in November 2008. During eight days, 48

127 random transects were performed by two observers in search of geckos under rocks (each 45

$128 \mathrm{~min}$, totalling $36 \mathrm{~h}$ of sampling) throughout the diel cycle. Temperatures of body (Tb, of the skin,

129 before touching the animal whenever possible or $<10 \mathrm{~s}$ after capture), soil under refuge (Ts) and

130 underside of rock used as refuge (Tr) were recorded (in shade if by daylight) with a Fluke ${ }^{\circ} 68$

131 infrared thermometer. Geckos tended to keep their original positions while Tb reading were

132 taken and only one rock was sampled at a time (tilting it to one side) to ensure that readings were

133 made as quickly as possible. Air temperature (Ta, correlated with humidity) was also measured

134 at $10 \mathrm{~cm}$ from the ground using a Fluke ${ }^{\circledR} 971$ temperature-humidity meter $\left( \pm 0.1{ }^{\circ} \mathrm{C}\right)$. Thermal

135 readings were made with the thermometer perpendicularly oriented to the surfaces and at very

136 close distances (10-20 $\mathrm{cm}$ of the animal) to ensure accurate measurements. The soil type was 
137 registered as compact or non-compact because this species avoids the latter (e.g. sandy soils)

138 (Schleich, 1987).

Quadrat sites were randomly chosen stratified by habitat types, with replicates across the

140 entire island according to habitat areas (Figure 1). The total area and percentage of cover of each

141 habitat registered on São Vicente, the number of quadrats per habitat, and the total and average

142 numbers of adults, juveniles and total number of geckos found in quadrats of each habitat are

143 detailed in Table S1. Sandy and saline areas were not sampled as geckos are absent from such

144 habitats (Schleich, 1987). In total, 40 quadrats of $10 \times 10$ meters were sampled in June 2010. All

145 geckos and refuge rocks available were counted within each quadrat. Rocks stuck to (or into) the

146 ground were not included, as they generally did not offer space for refuge. Sampling time of

147 quadrats varied according to gecko and refuge densities. As T. substituta was found to have

148 exclusively nocturnal activity (Vasconcelos, Santos \& Carretero, 2012), sampling of quadrats

149 was performed only during the day to maximize the probability of encountering inactive animals

150 under rocks and obtain the necessary data for calculating densities of geckos per quadrat.

151 Geckos detected under rocks along transects and quadrats were captured by hand, sexed,

152 photographed (for assigning sex in case of doubt), measured (SVL) for establishing the age

153 category and released at the capture site as quickly as possible to minimize stress. It was

154 registered whenever more than one animal was found under the same rock. Geckos were

155 classified as juveniles, adult males or adult females according to body size and sexual characters.

156 Refuges found to be used by geckos in transects and quadrats were categorised according to rock

157 size. Rock sizes were classified according to their width, length and height by the same observer

158 as small (less than one hand span) or medium-large - (more than one hand span). According to

159 previous work using data-loggers in rocks with different sizes and measuring $\mathrm{Ta}$, $\mathrm{Tr}$ and $\mathrm{Ts}$, a 
160 clear relation exists between rock sizes and thermal regimes of refuges along the day and $\mathrm{Tb}$

161 (Vasconcelos, Santos \& Carretero, 2012). Thus, small rocks were classified as low-quality and

162 medium-large rocks as high-quality refuges for the following analyses.

163

\section{Statistical procedures}

165 A chi square test was used to check if the proportion of male-female couples is significantly

166 higher than a random association between pairs of individuals independently of sex. Log-linear

167 analysis was used to test differences in the use of refuges by solitary and pairs of geckos.

168 Specifically we tested the association between three categorical variables in a multidimensional contingency table. The variables were refuge sharing (two classes: shared - whenever two or

170 more geckos used it - and non-shared - when just one individual used it), refuge type (two

171 classes: low and high-quality) and soil type (two classes: compact and non-compact). Log-linear

172 analysis uses a likelihood ratio chi-square statistic. The algorithm generates several models to 173 test interactions among all variables and selects the least complex model that best accounts for

174 the variance in the observed frequencies. Results were interpreted by checking odds-ratio scores

175 in expected values of partial and marginal association tests of variables retained in the model;

176 odd-ratio computes the likelihood ratio statistic of the model containing or not a particular term

177 (significant when $\mathrm{p}<0.05)$ (Jobson, 1992).

$178 \quad$ Values of $\mathrm{Tb}$ were compared between categories of adult geckos (solitary vs. aggregated)

179 with General Linear Models (GLM), using environmental and rock temperatures (Ta and $\mathrm{Tr}$ 180 respectively) as continuous predictors (i.e. covariates) to control their effect on $\mathrm{Tb}$, in order to

181 test if refuge sharing could influence $\mathrm{Tb}$ of the pair. Only one pair of geckos was found sharing 182 refuges during the night, so we have restricted our analyses to animals found during the day. 
183 Juveniles were excluded as refuge sharing was found in very few cases. For geckos sharing a 184 refuge, $\mathrm{Tb}$ was considered as the mean value of the two individuals, as $\mathrm{Tb}$ was found to be 185 independent of sex in a previous study (Vasconcelos, Santos \& Carretero, 2012). In this study, 186 the average difference between $\mathrm{Tb}$ of a pair was $1.18^{\circ} \mathrm{C} \pm 0.18$, and differences in $\mathrm{Tb}$ were again 187 not related to sex (Wilcoxon Matched Pair test $\mathrm{Z}=1.09, \mathrm{n}=29, \mathrm{p}=0.3$ ).

188 Non-parametric statistics were used whenever variables distribution violated normality 189 and homoscedasticity assumptions. Mann-Whitney U tests were performed to check whether 190 quadrats with geckos sharing a refuge had a different number of high-quality rocks than those 191 without geckos and to check if adult individuals that shared refuges presented significantly 192 higher SVL than those individuals that did not.

\section{Ethics statement}

License nr. 11/2008 was provided by Direcção Geral do Ambiente of Cabo Verde to perform

196

197

fieldwork in São Vicente that approved all sampling procedures. Geckos detected under rocks along transects and quadrats were captured by hand, sexed, photographed and measured as quickly as possible to minimize stress, and released at the capture site. No animals were killed or harmed. All animals were put in cloth bags during sampling to avoid double counts and to ameliorate stress.

\section{Results}

Aggregation incidence, size and composition

In total, 304 geckos were found under rocks (194 in transects and 110 in quadrats). Approximately 32 and 38\% of the geckos aggregated under rocks (i. e., were found in contact 
206 with or within the distance of their own body length from at least one other individual) in

207 transects and quadrats, respectively. Refuge sharing was detected 50 times (30 times in transects,

20820 in quadrats) in a total of 250 sampled refuges ( $20 \%$ of the sampled refuges), $94 \%$ of these by

209 a pair of individuals. Refuge sharing was observed between 48 male-female pairs of adults, one

210 male-male pair of adults, and one pair of juveniles. In three cases (two in transects and one in

211 quadrats) a juvenile was found sharing a refuge with a male-female pair. Given that the species

212 has a sex-ratio of near 1:1 (Vasconcelos, Santos \& Carretero, 2012), the proportion of male-

213 female pairs is significantly higher than a random association between pairs of individuals

214 independently of sex (chi-square test, $\chi 2=25.53 ; \mathrm{p}<0.001$ ). Adult sizes was not significant for

215 refugee sharing (Mann-Whitney $\mathrm{U}$ test, $\mathrm{Z}=6057, \mathrm{p}=0.0293 ; \mathrm{N}=203$ ). 


\section{Influence of ecological factors: gecko and refuge densities and refuge quality}

218 The log-linear analysis of frequency tables provided a good adjustment to the best model

219 (maximum likelihood ratio $\chi 2=1.30, \mathrm{df}=2, \mathrm{p}=0.52$ ) and retained the interactions between rock

220 size and refuge sharing in the best model (Table 1). The marginal table from which the odds-

221 ratio values were calculated showed that high-quality refuges (medium-large rocks) were shared

2225.4 times more frequently than low-quality refuges (small rocks). In contrast, non-shared refuges

223 were selected by single geckos in more similar frequencies, although high-quality refuges were

224 used 1.4 times more than low-quality ones.

225 A total of 110 geckos were found in quadrats (average density $\pm \mathrm{SD}=2.8 \pm 3.8$ geckos

226

227

228

229

230

231

232

233

234

235

236

237

238

239

per quadrat). We did not find differences in the densities of geckos across an altitudinal gradient within the commonest arid habitat on the island, i.e. flat, hilly and mountainous (Poisson distribution, $\log$ function, Wald statistic $\mathrm{W}=4.04, \mathrm{~N}=30 ; \mathrm{p}=0.13)$. Accordingly, gecko abundances in quadrats with different altitudes were pooled. Geckos were found in $73 \%$ of the 40 quadrats examined across the major habitats of the island. Quadrats with geckos $(\mathrm{N}=29)$ had a higher number of high-quality rocks than those without geckos $(\mathrm{N}=11)$ with marginal significance (Mann-Whitney $U$ test, $Z=1.73, p=0.08 ; N=40$ ). Refuge sharing was detected in nine of the quadrats with geckos, seven in arid habitats, and the number of shared refuges increased as more geckos were observed within a quadrat $(N=29$, Spearman $R=0.71, p<0.000$; Figure 2). This test was performed only in arid habitats to avoid a possible bias on densities in other habitat types. The number of high-quality refuges per gecko (average $\pm \mathrm{SD}=7.84 \pm 10.6$ ) was significantly smaller in those quadrats where we found refuge sharing $(2.3 \pm 1.8)$ than in those with no refuge sharing $(10.2 \pm 11.6)$, even when testing this only for arid habitats (MannWhitney $U$ test, $Z=-2.546, p=0.001 ; \mathrm{N}=29)$. 


\section{Influence of social factors: thermoregulation and mating}

242 Considering diurnal adult individuals observed under refuges, the GLMs did not detect

243 differences between $\mathrm{Tb}$ of geckos sharing or not sharing refuges once the effects of $\mathrm{Ta}$ and $\mathrm{Tr}$

244 were statistically accounted for $(\mathrm{F} 1,78=0.38, \mathrm{p}=0.54)$. The intersexual composition was

245 observed independently of the time of the year of the sampling, i.e. November and June (authors, 246 personal observations).

\section{Discussion}

This study showed that aggregations are common in T. substituta, with more than a third of the individuals found sharing refuges. It adds to anecdotal observations of aggregations in other aggregation in these cases are unknown (JC Brito, GV Antón, C Rato, R Ribeiro and J Teixeira, pers. obs.). Compared to other gecko species, aggregation in T. substituta is less common than in the Duvaucel's gecko Hoplodactylus duvaucelii, U. milli, and C. bibronii, where its proportion is between 47 to 71\% (Lemos-Espinal, Smith \& Ballinger, 1997; Shah, 2002; Mouton, 2011; Barry, Shanas \& Brunton, 2014). According to Mouton (2011), the nocturnal lifestyle of geckos, minimising predation pressure during activity, and the ground-dwelling nature of some, with individuals moving away from the shelter to forage, minimizing competition for food, are probably the key determinants allowing the occurrence of the aggregation behaviour. Such is the case in T. substituta, which was described as strictly nocturnal (Vasconcelos, Santos \& obs.). But, are these aggregations driven mainly by ecological or social factors? 
264 sites based on their thermal properties (Vasconcelos, Santos \& Carretero, 2012). Given its high

265 density and that its microhabitat choice is constrained mostly by the high diurnal temperatures,

266 we hypothesized that the tendency of individuals to aggregate was mostly related to high-quality

267 refuge scarcity (ecological aggregation) and not improved thermoregulation by physical contact

268 with conspecifics (thermal aggregation, a type of social aggregation). Our analyses showed that

269 even though high-quality refuges were used by both solitary and paired geckos in higher

270 frequencies than low-quality ones, they were shared much more frequently than for the latter.

271 They also showed that quadrats with the presence of geckos had a significantly higher number of

272 rocks than those without geckos. These results indicate that the presence of geckos is associated

273 to the presence of high-quality refuges, similarly to what was found to Egernia striolata skink

274 (Michael, Cunningham \& Lindenmayer, 2010). Moreover, the number of shared refuges

275 increased as more geckos were observed within a quadrat, indicating a strong correlation

276 between refuge sharing and density of conspecifics. Relationship between refuge sharing and

277 high-quality refuges availability was inverse, i.e. in those quadrats where we found cases of

278 refuge sharing the number of medium-large rocks per gecko was significantly smaller than in

279 those with no refuge sharing.

280 The GLMs did not detect differences between body temperatures of geckos sharing or not

281 sharing refuges during the day, once the effects of air and rock temperature were statistically

282 controlled. Thus, Tarentola substituta does not seem to improve thermoregulation by physical

283 contact with conspecifics, contrary to what happens in U. milii (Shah 2002). Tarentola substituta

284 seems thus to aggregate due to the beneficial thermal regime of high-quality refuges (that are

285 scarce) and not to improve thermoregulation by physical contact. Differences between U. milii 
286

287

288

289

290

291

292

293

294

295

296

297

298

299

300

301

302

303

304

305

306

307

308

and T. substituta can be the result of a difference in body size (the former is larger,

approximately $80 \mathrm{~mm}$ SVL as adults, and so body temperatures should naturally vary more

slowly), as well as environmental conditions (the former is subjected to much more variable

thermal regimes than those existing on São Vicente Island, and possibly the adaptive pressure for social thermoregulation is higher). However, the absence of more studies analysing the causes of aggregation in geckos does not allow generalizing patterns.

Interestingly, our study further shows that $T$. substituta aggregations are mainly

intersexual, as the proportion of male-female pairs is significantly higher than a random

association between pairs of individuals, similarly to what was found in H. duvaucelii (Barry,

Shanas \& Brunton, 2014). This suggests that social factors also play a role. There are few

examples of gecko intersexual aggregations, but these are usually composed of only one male

and one or more females (Chapple, 2003; Mouton, 2011; Barry, Shanas \& Brunton, 2014). The

most probable explanation of an intersexual aggregation, still to be experimentally tested, might

be to avoid agonistic interactions that potentially might result in fatal or debilitating

consequences for reptiles (Cooper \& Vitt, 1987). Intraspecific agonistic behaviour is common in

geckos, with a high level of intraspecific territoriality and associated male-male aggression

(Stamps, 1977). In contrast, male-female and female-female aggression are far less frequent

(Bolger \& Case, 1992; Petren \& Case, 1998; Dame \& Petren, 2006; Barry, Shanas \& Brunton, 2014). Higher levels of agonistic behaviour between males seems to be associated with marked sexual dimorphism, which generally is expressed by longer and heavier bodies, and wider heads in males than females (Bolger \& Case, 1992). In São Vicente Island, Tarentola substituta shows a marked sexual dimorphism (Vasconcelos, Rocha \& Harris 2012b) and high population densities, a common trend in island lizard populations (Whittaker \& Fernández-Palacios, 2007). 
309 These, coupled with the scarcity of high-quality refuges, would potentially lead to an increase of

310 male-male agonistic interactions. We speculate that intersexual sharing of thermally-better

311 refuges might be the solution to reduce agonistic interactions, similarly to what has been

312 observed with Peers' girdled lizard Namazonurus peersi (Mouton, 2011).

313 Moreover, other factors such as enhanced chances for reproduction could favour

314 intersexual aggregations. For example, male Eublepharis macularius geckos previously housed

315 with females displayed more territorial and courtship behaviour relative to males housed in

316 isolation (Sakata et al., 2002). Thus, it is possible that males of T. substituta prefer to share high-

317 quality refuges with adult females not only to reduce the probability of aggressive agonistic

318 interactions because they are generally less aggressive, but also to increase the probability of

319 mating. That we found this intersexual composition in two different times of the year (November

320 and June and confirmed this with ad hoc observations in February, April and May in different

321 years) supports some stability of aggregations in this species and dilutes the strength of the

322 alternative hypothesis of explain them as fortuitous mating encounters, even though the precise

323 reproductive period of the species is still unknown. A common feature of stable aggregation

324 reported by Gardner et al. (2015) was the occurrence of juveniles with adults of either or both

325 sexes, as we observed in this study. Further support comes from the observation that the

326 juveniles found together with those pairs were not recently born and from other gecko species

327 with similar trends (Barry, Shanas \& Brunton, 2014). Those juveniles may be tolerated by adults

328 because they pose no threat to their reproduction or present any inbreeding risk (see Gardner et

329 al., 2015).

Another question that can be raised is why female-female T. substituta couples do not occur more frequently if agonistic interactions are also low? Dame \& Petren's (2006) 
332 experiments have proved that the presence of males affected foraging of females: Hemidactylus

333 frenatus males consumed fewer resources and consistently turned their attention towards

334 courtship when females were present (Dame \& Petren, 2006). Following the same authors, the

335 reduced foraging effort by males probably leads to increased foraging opportunities for females,

336 who may increase their resource intake to increase provisioning of eggs in the advent of

337 fertilization. It remains to be explored if this applies to T. substituta system as well.

338 Aggregation behaviour has evolved repeatedly in groups of reptiles that are mainly

339 solitary, and pair- or kin-bonds are a simple form of socialization that may appear under certain

340 circumstances, such as refuge scarcity (Chapple 2003; Gardner et al 2015). Cases such as $T$.

341 substituta, suggest that this behavioural plasticity may ease the evolution of more complex social

342 behaviours in solitary taxa and supports the idea of broader sociality incidence for reptiles

343 (Doddy et al. 2012). This is amongst the first documented evidence of aggregation behaviour in

344 geckos of the Phyllodactylidae family, and it suggests differences from the scenario suggested by

345 Gardner et al. (2015). Many questions related to the determinants and consequences of this

346 aggregation behaviour remain unanswered. For example, we do not know whether male-female

347 pairs actually result in higher mating/reproduction success, and whether those pairs are

348 monogamous associations or not. Monogamous or near monogamous mating systems are known

349 in lizards but are poorly documented - they seem to be the case in Tiliqua rugosa (Bull \&

350 Baghurst, 1998), Egernia skinks (Chapple, 2003) and Gymnodactylus geckos (Pianka \& Vitt,

351 2003). Reptile monogamy seems to be more common in long-lived species that produce few

352 descendants, have high parental investment and high survivorship of adult and juveniles (Pianka

$353 \&$ Vitt, 2003). Unfortunately, basic ecological data are not available for any of the Cape Verde

354 Tarentola species (Schleich, 1987; Vasconcelos et al., 2013). New studies aimed at disentangling 
355 basic ecological trends in reptiles from Cabo Verde would be fruitful to understand ecological

356 processes of islands reptiles and to formulate guidelines for their conservation.

357

\section{Conclusion}

359

360

361

362

\section{References}

In summary, aggregation behaviour in T. substituta is common and seems to be mainly led by ecological (extrinsic) factors, i.e., scarcity of high-quality refuges coupled with high gecko densities. This species seems to aggregate to benefit from the thermal regime of high quality refuges and not to improve thermoregulation by physical contact. Aggregations mostly involve male-female pairs, possibly to avoid agonistic interactions, but perhaps also to increase mating opportunities. Several aspects of the determinants and consequences of this aggregation behaviour could be further characterized, such as aggregation stability.

\section{Acknowledgments}

We thank to Cabeólica S.A. for logistical support. We thank Gonçalo Cardoso and previous reviewers for helpful suggestions. We thank John Archer for reviewing the English.

Alexander RD. 1974. The evolution of social behaviour. Annual Review of Ecology and Systematics 5:325-383.

Atzori A., Berti F., Tamburini M., Zuffi M., Fornasiero S., Cencetti T. 2007. Advances in methodologies of sexing and marking less dimorphic gekkonid lizards: the study case of the Moorish gecko, Tarentola mauritanica. Amphibia-Reptilia 28:449-454. DOI:
$10.1163 / 156853807781374719$. 
378 Barry M., Shanas U., Brunton DH. 2014. Year-round mixed-age shelter aggregations in

379 Duvaucel's geckos (Hoplodactylus duvaucelii). Herpetologica 70:395-406. DOI: 10.1655/HERPETOLOGICA-D-13-00056.

381

382

Bauer AM. 1990. Phylogenetic systematics and biogeography of the Carphodactylini (Reptilia: Gekkonidae). Bonner zoologische Monographien 30:1-220.

Bolger DT., Case TJ. 1992. Intra- and interspecific interference behaviour among sexual and asexual geckos. Animal Behaviour 44:21-30. DOI: 10.1016/S0003-3472(05)80750-X.

Bull C., Baghurst B. 1998. Home range overlap of mothers and their offspring in the sleepy lizard, Tiliqua rugosa. Animal Behaviour 42:357-362.

Chapple DG. 2003. Ecology, life-history, and behavior in the australian scincid genus Egernia, with comments on the evolution of complex sociality in lizards. Herpetological Monographs 17:145. DOI: 10.1655/0733-1347(2003)017[0145:ELABIT]2.0.CO;2.

Cooper WE., Vitt LJ. 1987. Deferred agonistic behavior in a long-lived scincid lizard Eumeces laticeps. Oecologia 72:321-326. DOI: 10.1007/BF00377558.

Dame E a., Petren K. 2006. Behavioural mechanisms of invasion and displacement in Pacific island geckos (Hemidactylus). Animal Behaviour 71:1165-1173. DOI: 10.1016/j.anbehav.2005.10.009.

Davis AR., Corl A., Surget-Groba Y., Sinervo B. 2011. Convergent evolution of kin-based sociality in a lizard. Proceedings of the Royal Society B: Biological Sciences 278:15071514. DOI: $10.1098 /$ rspb.2010.1703.

Doody JS., Burghardt GM., Dinets V. 2013. Breaking the Social-Non-social Dichotomy: A Role for Reptiles in Vertebrate Social Behavior Research? Ethology 119:95-103. DOI: 10.1111/eth.12047. 
401 Gardner MG., Pearson SK., Johnston GR., Schwarz MP. 2015. Group living in squamate 402 reptiles: a review of evidence for stable aggregations. Biological Reviews:n/a-n/a. DOI: 10.1111/brv.12201.

404 Graves BM., Duvall D. 1995. Aggregation of Squamate Reptiles Associated with Gestation, 405 Oviposition, and Parturition. Herpetological Monographs 9:102. DOI: 10.2307/1466999. 406 Greenberg B. 1943. Social behavior of the western banded gecko, Coleonyx variegatus Baird. $407 \quad$ Physiological Zoology 16:110-112.

408 Hijmans RJ., Cameron SE., Parra JL., Jones PG., Jarvis A. 2005. Very high resolution 409 interpolated climate surfaces for global land areas. International Journal of Climatology 25:1965-1978. DOI: 10.1002/joc. 1276.

411

412

413

414

415

416

417

418

419

420

421

422

423

Jobson J. 1992. Applied Multivariate Data Analysis Vol II: Categorical and Multivariate Methods. New York: Springer-Verlag.

Joger U. 1984. Taxonomische Revision der Gattung Tarentola (Reptilia: Gekkonidae). Bonner zoologische Beiträge 35:129-174.

Kearney M., Shine R., Comber S., Pearson D. 2001. Why Do Geckos Group? An Analysis of “Social” Aggregations in Two Species of Australian Lizards. Herpetologica 57:411-422. DOI: 3893048 .

Lancaster JR., Wilson P., Espinoza RE. 2006. Physiological benefits as precursors of sociality: why banded geckos band. Animal Behaviour 72:199-207. DOI: 10.1016/j.anbehav.2006.01.010.

Lemos-Espinal JA., Smith GR., Ballinger RE. 1997. Natural history of Xenosaurus platyceps, a crevice dwelling lizard from Tamaulipas, Mexico. Herpetological Natural History 5:181186. 
424 Martín J., Polo-Cavia N., Gonzalo A., López P., Civantos E. 2011. Social aggregation behaviour 425 in the North African amphisbaenian Trogonophis wiegmanni. African Journal of Herpetology 60:171-176. DOI: 10.1080/21564574.2011.566285.

427

428

429

430

431

432

433

434

435

436

437

438

439

440

441

442

443

444

445

446

Meyer A., Mouton PLFN. 2007. Aggregation in Bibron's gecko, Chondrodactylus bibronii. African Journal of Herpetology 56:137-147. DOI: 10.1080/21564574.2007.9635559.

Michael DR., Cunningham RB., Lindenmayer DB. 2010. The social elite: Habitat heterogeneity, complexity and quality in granite inselbergs influence patterns of aggregation in Egernia striolata (Lygosominae: Scincidae). Austral Ecology 35:862-870. DOI: 10.1111/j.14429993.2009.02092.x.

Mouton PLFN. 2011. Aggregation behaviour of lizards in the arid western regions of South Africa. African Journal of Herpetology 60:155-170. DOI: 10.1080/21564574.2011.562926.

Panov EN., Zykova LY. 2003. Mountain agamas of Eurasia (Gornye agamy Evrazii). Moscow: Lazur.

Petren K., Case TJ. 1998. Habitat structure determines competition intensity and invasion success in gecko lizards. Proceedings of the National Academy of Sciences 95:1173911744. DOI: $10.1073 /$ pnas.95.20.11739.

Pianka E., Vitt L. 2003. Lizards: windows to the evolution of diversity. Berkeley, Los Angeles, London: University of California press.

Ruby DE. 1977. Winter activity in Yarrow's spiny lizard, Sceloporus jarrovi. Herpetologica $33: 322-333$.

Sakata JT., Gupta A., Chuang C-P., Crews D. 2002. Social experience affects territorial and reproductive behaviours in male leopard geckos, Eublepharis macularius. Animal Behaviour 63:487-493. DOI: 10.1006/anbe.2001.1952. 
447 Schleich H-H. 1987. Herpetofauna Caboverdiana. Spixiana 12:1-75.

448 Shah B. 2002. Why do thick-tailed geckos (Underwoodisaurus milii) aggregate? University of 449 Sydney, Sydney, New South Wales, Australia.

450 Shine B., Shah R., Hudson S., Kearney M. 2003. Sociality in Lizards: Why Do Thick-tailed 451 Geckos (Nephrurus milii) Aggregate? Behaviour 140:1039-1052. DOI:

452 $10.1163 / 156853903322589632$.

453 Stamps J. 1977. Social behavior and spacing patterns in lizards. In: Gans C, Tinkle DW eds. 454 Biology of the Reptilia. London: Academic Press, 265-334.

455 Toxopeus AG., Krujit JP., Hillenius D. 1988. Pair-bonding in chameleons. Naturwissenschaften $456 \quad 75: 268-269$.

457 Vasconcelos R., Brito JC., Carranza S., Harris DJ. 2013. Review of the distribution and 458 conservation status of the terrestrial reptiles of the Cape Verde Islands. Oryx 47:77-87. 459 DOI: $10.1017 / \mathrm{S} 0030605311001438$.

460 Vasconcelos R., Perera A., Geniez P., Harris DJ., Carranza S. 2012. An integrative taxonomic 461 revision of the Tarentola geckos (Squamata, Phyllodactylidae) of the Cape Verde Islands. 462 Zoological Journal of the Linnean Society 164:328-360. DOI: 10.1111/j.10963642.2011.00768.x.

464

Vasconcelos R., Rocha S., Harris DJ. 2012. Environmental impact assessment of the S. Vicente 465 wind farm on the Tarentola caboverdiana substituta gecko - final report. CIBIO-InBIO.

Vasconcelos R., Santos X., Carretero MA. 2012. High temperatures constrain microhabitat 467 selection and activity patterns of the insular Cape Verde wall gecko. Journal of Arid Environments 81:18-25. DOI: 10.1016/j.jaridenv.2012.01.013.

469 Vitt LJ., Zani PA., Caldwell JP. 1996. Behavioural ecology of Tropidurus hispidus on isolated 
470 rock outcrops in Amazonia. Journal of Tropical Ecology 12:81-101. DOI:

$471 \quad 10.1017 / \mathrm{S} 0266467400009329$.

472 Whittaker RJ., Fernández-Palacios JM. 2007. Island Biogeography - Ecology, evolution, and

473 conservation. Oxford, New York: Oxford University Press.

474 Wilson EO. 1975. Sociobiology, the New Synthesis. Cambridge, UK: Belknap Press of Harvard $475 \quad$ University Press.

476 


\section{Table $\mathbf{1}$ (on next page)}

Results of the log-linear analysis of interaction between refuge sharing, refuge type and soil type.

Results of the log-linear analysis indicating the values of the partial and marginal association tests between the three variables. The informative results come from the interactions between the two environmental variables (refuge quality, RQ and soil type, ST) and refuge sharing, share, with the classes 'sharing' and 'non-sharing'. df: degrees of freedom; $p$ : $p$ value. The values of the best model are marked in bold. 
1 Table 1.

\begin{tabular}{lccccc}
\hline & d & Partial $\chi \mathbf{2}$ & $\mathbf{p}$ & Marginal $\chi^{2}$ & $\mathbf{p}$ \\
\hline RQ & 1 & 12.43 & 0.0004 & 12.43 & 0.0004 \\
ST & 1 & 60.87 & $<0.0001$ & 60.87 & $<0.0001$ \\
share & 1 & 66.42 & $<0.0001$ & 66.42 & $<0.0001$ \\
RQ x ST & 1 & 5.700 & 0.020 & 7.080 & 0.008 \\
RQ x share & $\mathbf{1}$ & $\mathbf{6 . 8 2 0}$ & $\mathbf{0 . 0 0 9}$ & $\mathbf{8 . 1 9 0}$ & $\mathbf{0 . 0 0 4}$ \\
ST x Ref & 1 & 1.170 & 0.280 & 2.550 & 0.110 \\
\hline
\end{tabular}

2 
Figure 1

Study area and study site.

Map of the Cabo Verde Islands showing the location of the archipelago relative to the West

African coast (A), its elevations and the location of São Vicente Island (B), and of the

sampling sites (C), either transects (rectangle) and quadrats (circles); Geographic Coordinate

System, Datum WGS84. Mapped habitats (São Vicente) are adapted from Diniz \& Matos
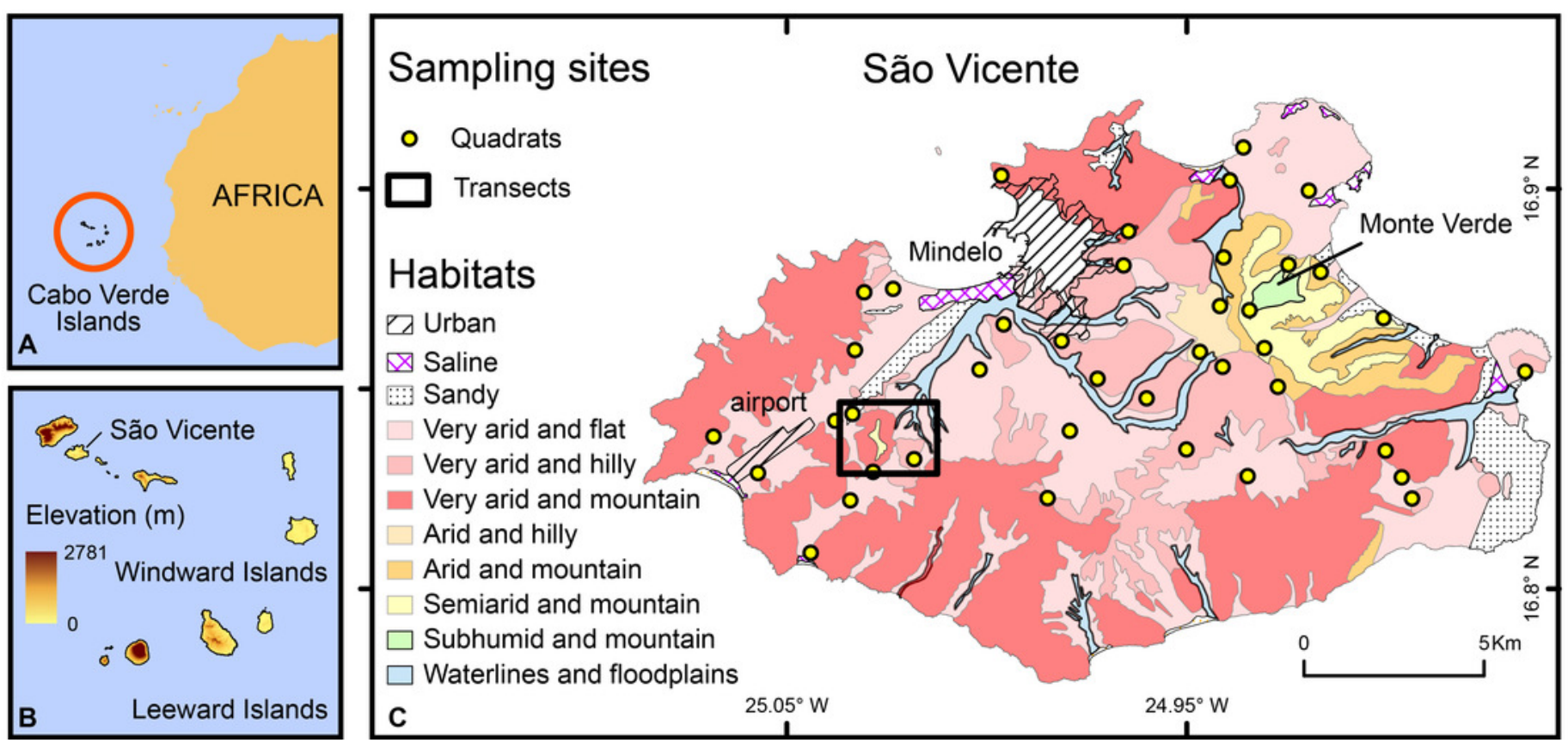
Figure 2 (on next page)

Relationship between refuge sharing and gecko densities on São Vicente Island.

Refuge sharing is given by the number of shared refuges detected per quadrat and gecko densities by the total number of geckos per quadrat. The Spearman correlation value is also given (R). Values with overlapping points in grey. $N=29$ quadrats with geckos ( 9 with geckos sharing refuges and 20 with single geckos). 


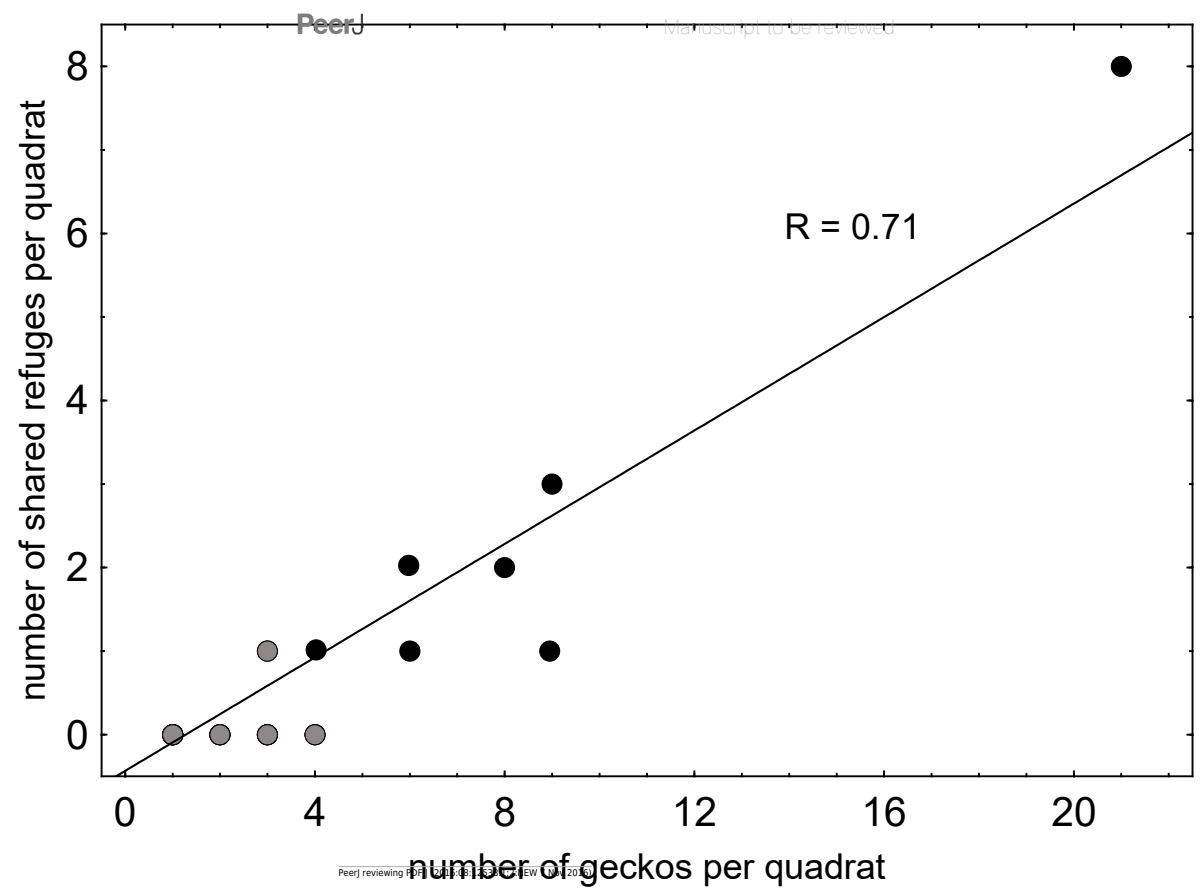

